\title{
Neumomediastino espontáneo idiopãtico. Primer caso pediátrico en Colombia
}

\author{
Jessica Natalia Archila - Diaz * \\ Jenifer Walteros - Cárdenas ** \\ Víctor Manuel Mora - Bautista ***
}

*Médica UNAB. Médico urgencias Clínica Materno Infantil San Luis. Bucaramanga, Santander - Colombia. **Médica UDES. Médico urgencias Clínica Materno Infantil San Luis. Bucaramanga, Santander - Colombia.

***Médico y cirujano UIS. Especialista en pediatría UIS. Pediatra urgencias Clínica Materno Infantil San Luis. Bucaramanga, Santander - Colombia. Correspondencia: Dr. Víctor Manuel Mora Bautista. Dirección: Calle 4825 - 56. Barrio Nuevo Sotomayor. Clínica San Luis. Teléfono: 3045321812. Correo electrónico: vmoramd@medicos.com

\section{Resumen}

El neumomediastino espontáneo idiopático es la presencia de aire en el mediastino, sin ninguna causa aparente, siendo descrito en 1939 por el Dr. Hamman. Se atribuye a una fuga dinámica de aire desde los alvéolos a través del intersticio de personas jóvenes (efecto Macklin). La clínica no es específica, pero el dolor torácico agudo retroesternal y la tos, son los síntomas más orientativos; los signos más específicos son los crépitos precordiales (signo de Hamman) y el enfisema subcutáneo. La radiografía de tórax es suficiente para el diagnóstico, aunque otras técnicas de imagen sirven para evaluar causas secundarias. El tratamiento es reposo, analgésicos y, opcionalmente, oxígeno suplementario. Se presenta el caso de un niño en edad escolar con neumomediastino espontáneo sin ningún desencadenante identificable, siendo el primer caso pediátrico colombiano reportado de esta variante. MÉD.UIS.2019;32(2):47-52

Palabras clave: Neumomediastino. Dolor torácico. Enfisema subcutáneo

\section{Idiopathic spontaneous pneumomediastinum. First colombian pediatric case.}

Abstract

Idiopathic spontaneous pneumomediastinum is the presence of air in the mediastinum, without any apparent cause, it was described in 1939 by Dr. Hamman. It is attributed to a dynamic escape of air from the alveoli through the pulmonary interstice of young people (Macklin effect). The clinical symptoms are not specific, but acute retrosternal chest pain and cough are the most indicative symptoms; the most specific signs are precordial crepitus (Hamman's sign) and subcutaneous emphysema. The chest X-ray is enough for the diagnosis; however, other imaging techniques are used to evaluate secondary causes. The treatment is rest, analgesics and, optionally, supplemental oxygen. There is presented the case of a school-age child with spontaneous pneumomediastinum without any identifiable trigger, being the first reported Colombian pediatric case of this variant. MÉD.UIS.2019;32(2):47-52

Keywords: Pneumomediastinum. Chest pain. Subcutaneous emphysema.

¿Cómo citar este artículo?: Archila-Díaz JN, Walteros-Cárdenas J, Mora-Bautista VM. Neumomediastino espontáneo idiopático. Primer caso pediátrico en Colombia. MÉD. UIS.2019;32(2):47-52. doi: 10.18273/revmed.v32n2-2019006 


\section{Introducción}

Neumomediastino es la presencia de aire en el espacio mediastínico, que puede ocurrir por cuerpos extraños, episodios eméticos, traumatismos, accesos de tos, otras maniobras de Valsalva, hiperventilación, procedimientos odontológicos con extracción dental, inmersiones, entre otras ${ }^{1-5}$. Es una enfermedad infrecuente, que se diagnostica en $1 / 1000-44000$ ingresos hospitalarios en series internacionales $s^{4,6-8}$. No existen datos sobre frecuencia de presentación en Colombia, aunque hay un caso reportado por Morcillo y Vallejo (2015) en un adolescente de 15 años que practicaba motocross y ejercicio de carga pesada². Otro caso colombiano, fue reportado por Estrada et al. (2009) en una adolescente de 12 años que levantaba sacos de tierra 3. Su edad de presentación es variable, aunque de predominio en adultos jóvenes, y la mayoría de los casos son de sexo masculino $0^{4,5,8,9}$.

La clínica característica es dolor torácico agudo retroesternal que aumenta con los movimientos respiratorios y la tos aunque en ocasiones se acompaña de disnea. Sólo hasta la aparición del signo de Hamman (crujidos o crepitantes precordiales) y el enfisema subcutáneo cervical o precordial, es que el diagnóstico clínico se hace muy evidente, reflejando la complejidad semiológica del neumomediastino, dada la baja frecuencia de estos signos (alrededor de $30 \%$ de los casos). Debido a lo anterior, la clave diagnóstica puede ser sólo la radiología ${ }^{6,10,11}$.

El nombre de síndrome de Hamman viene de la descripción en 1939 por Louis Virgil Hamman del enfisema mediastínico espontáneo $0^{6,9}$. Actualmente se atribuye esta enfermedad a fuga dinámica de aire desde los alvéolos hacia el mediastino a través del intersticio peribroncovascular, el cual es laxo en los pacientes jóvenes, sin romper la pleura visceral (efecto Macklin) $)^{3,12,13}$.

La primera controversia en esta patología es establecer su naturaleza espontánea. Puede corresponder hasta un 40 - 50\% de todos los neumomediastinos $\mathrm{s}^{2,4,8,14-16}$. La incidencia no es precisa y cambia dependiendo si se incluyen los factores precipitantes como causas secundarias o no (Ver Figura 1), aunque la evolución clínica es similar ${ }^{11}$.

Los cirujanos tienen competencia en la patología, dado que participan del diagnóstico diferencial descartando las causas secundarias más graves (ruptura tráqueo-bronquial, perforación esofágica, mediastinitis, etc. $)^{6}$.

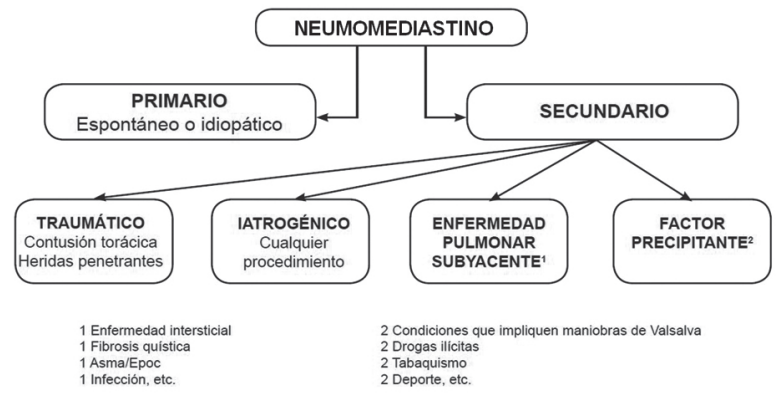

Figura 1. Clasificación del neumomediastino. Fuente: Campbell et al. 2016 $^{16}$.

Suele presentarse como neumomediastino aislado (50\%) o asociado a enfisema subcutáneo (37\%); los casos restantes corresponden a formas debutantes con enfisema subcutáneo o asociadas a neumotórax ${ }^{4}$.

Independiente de la edad de presentación, se han descrito varios signos en la radiografía simple de tórax, entre los que destacan el neumopericardio, el signo de la $V$ de Naclerio (presencia de aire entre la aorta descendente y el hemidiafragma izquierdo), el resalte del contorno cardíaco (por la presencia de aire mediastínico) y el aire delimitando el timo (signo de la vela $)^{6,17}$. Entre otras técnicas diagnósticas, la tomografía computarizada o el estudio esofágico están indicadas en pacientes con importante compromiso respiratorio y/o hemodinámico, o ante la sospecha de perforación esofágicaa ${ }^{18-20}$.

Aún hay controversia sobre si se debe realizar siempre la tomografía. Podría ser prudente no realizar más exámenes en los niños sin causa aparente luego de una anamnesis detallada, sin eventos de atoramiento, e incluso a los que cursan con asma, por su evolución benigna ${ }^{15-17,21-23}$. El ecocardiograma podría ser de utilidad y es más accesible que la tomografía; el signo representativo en esta técnica es la brecha de aire, donde la acumulación de aire no deja visualizar el corazón en la proyección apical y paraesternal ${ }^{24,25}$.

La evolución tiende a la resolución espontánea en 2 15 días sólo con manejo conservador, salvo los casos que cursan con neumotórax (pueden tardar más tiempo y debe insistirse más en buscar una causa secundaria) y las recurrencias (ocurren en menos de $5 \%$ ). Este abordaje consiste en reposo, analgesia y evitar maniobras que aumenten la presión 
pulmonar ${ }^{16,23,26}$; un tratamiento complementario es la terapia con altas concentraciones de oxígeno, aunque no es indispensable si el paciente mantiene una adecuada oxigenación sin dificultad respiratoria ${ }^{10,17,27}$. Ocasionalmente se recomienda iniciar tratamiento antibiótico para evitar infecciones ${ }^{1,12}$.

Se presenta el caso de un niño escolar con síndrome de Hamman a quien se le realizaron estudios imagenológicos y serológicos, descartándose una causa secundaria y exhibiendo una resolución rápida sólo con tratamiento médico. Se busca promover el reconocimiento adecuado de la entidad mediante la revisión de la semiología relacionada, dada la dificultad diagnóstica que acarrea establecer la naturaleza primaria del neumomediastino y la inexistencia de reportes previos pediátricos de esta variante en Colombia ${ }^{2,3,28}$.

\section{Reporte de caso}

Se presenta el caso de un niño de 9 años sin antecedentes médicos de importancia, quien inició de manera súbita (se encontraba en reposo en su casa), con accesos de tos seca metálica, disnea y dolor torácico intermitente en región retroesternal. Los síntomas ocurrieron concomitantemente, persistieron durante el día y no hubo desencadenante identificable.

Consultó el día en que iniciaron los síntomas al servicio de urgencias en su hospital local, siendo interpretada su sintomatología como laringitis obstructiva y recibiendo tres nebulizaciones con 1 mg de epinefrina, con poca mejoría al egresar ese mismo día. Persistió sintomático y reconsultó a urgencias al día siguiente, por lo que le realizaron un electrocardiograma que mostró un trazado normal y fue mantenido en observación. Al siguiente día presentó dolor torácico constante que aumentaba con el movimiento y apareció odinofagia. Fue evaluado por médico general, encontrándosele crépitos bifásicos en hemitórax izquierdo y ruidos interpretados como frote pleural; por este motivo se sospechó derrame pericárdico asociado a posible neumonía, siendo remitido para evaluación por pediatría en tercer nivel de atención.

Al ingreso a urgencias de pediatría, en su tercer día de enfermedad, no se documentó tos ni estridor; sin embargo, se auscultaron crépitos en región precordial, se documentó dolor en la región cervical anteroinferior y se palparon crepitaciones en esa zona. Ante estos hallazgos se realizó una radiografía de tórax evidenciando enfisema subcutáneo en cuello y neumomediastino global, por lo que se hospitalizó sospechando perforación esofágica versus ruptura de vía aérea. Se inició ampicilina sulbactam que se sostuvo por 7 días.

En ese mismo día, se obtuvo una tomografía de cuello y tórax observando neumomediastino que comprometía las tres regiones mediastinales (anterior, media y posterior) y se extendía a tejidos blandos del cuello con enfisema subcutáneo supraclavicular laterocervical derecho y en zona paraesternal (Ver Figura 2).

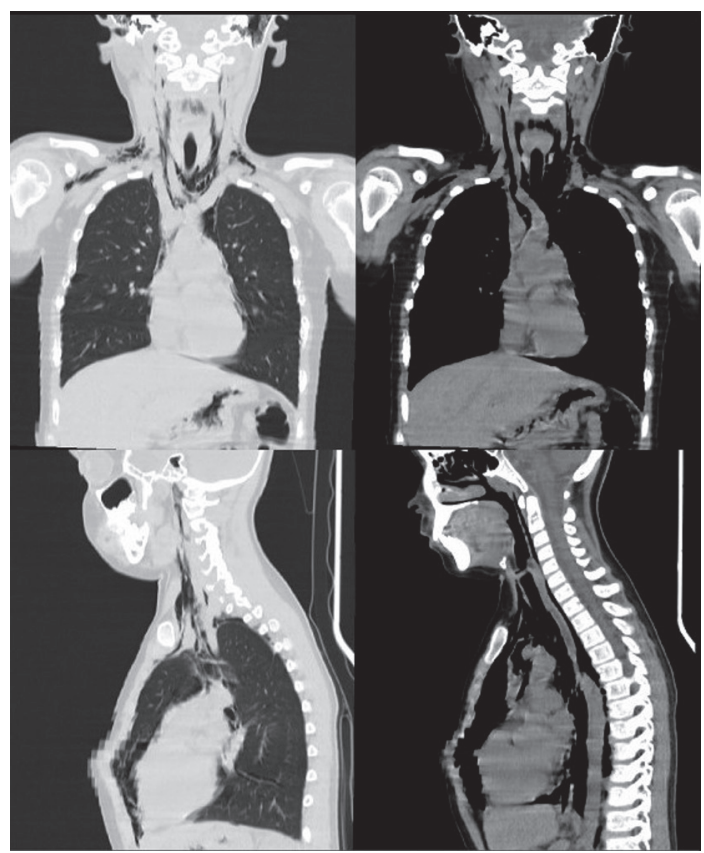

Figura 2. Tomografía cérvico-torácica.

Fuente: autores

Posteriormente, se le realizaron controles radiográficos durante la primera semana de estancia con disminución del neumomediastino y enfisema (Ver Figura 3). En el día primero se observaron imágenes radiolúcidas, que separaban la silueta cardiaca de una línea radiopaca que corresponde al pericardio separado por la presencia de aire tanto en la proyección AP como lateral. En los días siguientes desaparecieron gradualmente los hallazgos.

Cirugía pediátrica valoró tras 12 horas luego del ingreso a tercer nivel de complejidad y consideró manejo conservador con oxigenoterapia de alto flujo. En el quinto día de enfermedad se le practicó 
nasofibrolaringoscopia por otorrinolaringología, con resultado normal. Neumología valoró en el sexto día de enfermedad e indicó tomar Alfa-1-antitripsina, con reporte dentro de límites normales $(138 \mathrm{mg} / \mathrm{dl})$, y descartar linfangioleiomiomatosis como causas de enfermedad pulmonar intersticial que ocasionaran la fuga de aire. Ante esta sospecha diagnóstica, por indicación de gastroenterología, se le realizó ecografía abdominal (noveno día de enfermedad) y una esófagogastroduodenoscopia (duodécimo día de enfermedad), con reportes normales. Ante reporte endoscópico normal, tomografía de tórax no compatible, sexo masculino y grupo etario no frecuente, se excluyó la linfangioleiomiomatosis. Finalmente, no se identificó ningún desencadenante del neumomediastino, por lo que se estableció el diagnóstico de síndrome de Hamman y no se consideró requerido hacer más exámenes fuera de los controles radiológicos.

Egresó al décimo día de estancia (décimo tercero de enfermedad). Un mes después asistió a consulta con neumología pediátrica, con examen físico y radiografía de tórax normales, por lo que fue dado de alta.

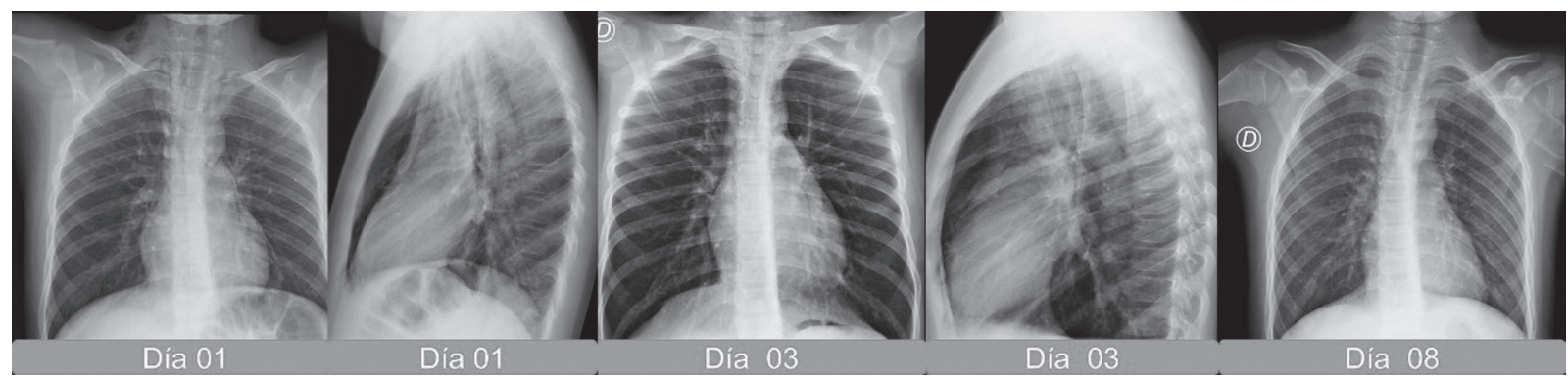

Figura 3. Radiografías realizadas durante la estancia. Fuente: autores

\section{Discusión}

En el caso expuesto, la sintomatología hizo pensar inicialmente a medicina general en laringitis obstructiva dadas las características de la tos (seca y en accesos), aunque la presencia y empeoramiento del dolor torácico no se correlacionaba bien con dicho diagnóstico. También es de resaltar que el dolor no precedió a la tos, sino que fueron concomitantes. Posteriormente en tercer nivel, no se encontraron signos compatibles con laringitis durante toda la estancia.

Dentro de lo conocido, el grupo etario del paciente presentado no es el más usual para las formas primarias de la patología (ocurren en adolescentes y adultos jóvenes), lo que hace aún más mandatorio buscar posibles causas secundarias ${ }^{4,28}$.

Para configurar el diagnóstico de síndrome de Hamman se requiere siempre una evaluación multidisciplinaria para descartar las causas secundarias. Se presenta la clasificación etiológica conocida (Ver Figura 1), con el propósito de diferenciar el neumomediastino primario o idiopático de todas las causas secundarias que se han reportado en la literatura. De ese flujograma se puede concluir que en el caso expuesto se lograron excluir todas las etiologías alternas.
La primera de dichas etiologías fue la laringitis obstructiva o crup, que fue descartada por la ausencia de semiología compatible y al no contar con historial de alergias respiratorias; la presencia de laringo o traqueomalacia se excluyó con la nasofibrolaringoscopia; la perforación por cuerpo extraño se excluyó al realizar evaluación endoscópica y tomográfica, dado que no siempre la ingestión del cuerpo extraño es referida claramente dentro de la anamnesis; el déficit de alfa-1-antitripsina se asocia con la presencia de enfermedad intersticial y bulas, que no fueron evidentes a nivel tomográfico; y la linfangioleiomiomatosis se origina por crecimiento anormal de células musculares lisas en el intersticio pulmonar principalmente pero afecta más a mujeres en edad fértil y también requiere una tomografía de tórax con compromiso intersticial. Existe una forma asociada a esclerosis tuberosa, por lo que la endoscopia digestiva ayudaba al diagnóstico encontrando masas, lo cual no sucedió en el caso presentado. A partir de la anamnesis, el examen físico y los laboratorios no había más etiologías alternas por descartar ${ }^{6,22,29,30}$.

A nivel mundial, el reporte más reciente es el de Almeida et al. (2017), quienes reportaron un caso en un niño de 3 años evaluado con radiografía y tomografía, confirmando el diagnóstico mediante 
radiografía de tórax y tomografía; recibiendo tratamiento con reposo y oxigenoterapia a alto flujo, documentando resolución en menos de una semana, aunque sin evaluarse adecuadamente para otras patologías secundarias ${ }^{26}$.

De la misma forma, se encuentran Gasser \& cols. (2016), quienes encontraron 216 pacientes descritos entre 1986 - 2011 y mencionan una edad promedio entre $7-14$ años junto con un $33 \%$ de casos como idiopáticos, ocurriendo el signo de Hamman en el $11.6 \%$ y el enfisema subcutáneo en el $66.4 \%$ (a nivel cervical profundo en el 50\%). Describieron un abordaje diagnóstico y terapéutico similar al presentado en el caso expuesto, con esofagogramas y nasofibrobroncoscopias realizadas según la anamnesis y la semiología. Además se empleó oxigenoterapia de alto flujo con todos los pacientes. Anotan que la mayoría de causas secundarias se identificaron en niños menores de 6 años $^{8}$.

En tercer lugar, Abbas \& cols. (2015), realizaron una revisión de casos de neumomediastino entre 2011 - 2014, incluyendo 129 niños entre 7 - 16 años, encontrando como predominantes los síntomas de dolor torácico (59\%), disnea (40\%) y tos (34\%); con ocurrencia de neumomediastino aislado en $50 \%$ de todos los casos; y clasificación final como casos espontáneos en un 53\%, luego de excluir los casos con maniobras de Valsalva (19\%). Es llamativo que no reportan la frecuencia de los signos clínicos (Hamman o enfisema), y el bajo uso requerido de tomografías (1.5\%). Reportan recurrencias entre $2-9$ meses en un $3 \%$ de los $\operatorname{casos}^{4}$. Este estudio resalta la sintomatología usual de esta patología y promueve la actitud conservadora en la imagenología empleada.

Por su parte, Zachariah \& cols. (2015), presentaron un caso de un adolescente de 17 años manejado con el mismo enfoque anterior donde sólo realizaron radiografía y ecocardiograma para corroborar el diagnóstico ${ }^{24}$. Kin-sun \& cols. (2013), expusieron 87 casos de neumomediastino en niños con sólo $32 \%$ de casos idiopáticos; los desencadenantes comunes fueron asma, infecciones respiratorias o atragantamientos ${ }^{15}$. Lee \& cols. (2010), describieron 37 casos presuntivos como espontáneos, entre 1994 - 2007, sólo con un $14 \%$ de estos correspondiendo a la verdadera forma espontánea, sin factores precipitantes, resaltan también que en preescolares es más común que haya causas secundarias ${ }^{14}$.

La serie de Álvarez \& cols. (2009), reportó sólo 8 casos de neumomediastino entre 16 - 55 años (promedio 25 años), con dolor torácico y enfisema subcutáneo como síntoma y signos predominantes, respectivamente; reportan el signo de Hamman sólo en 2 casos. Es de anotar que realizaron tomografía en 7 casos. Sin embargo, se desestima su relevancia, dado que todos los casos tenían un factor precipitante ${ }^{6}$.

Cho \& Kim (2010), estudiaron dos casos, un adolescente de 16 años y una adulta joven de 19 años, que presentaron clínica de dolor torácico, disnea y radiografía compatible con tomografía que confirmó el neumomediastino, resolviendo en 48 y 96 horas respectivamente ${ }^{20}$.

Crespo \& cols. (2006), mostraron un caso de un adolescente de 14 años con clínica similar al caso expuesto en esta investigación, sin precipitantes, pero evaluado sólo con radiografía y con resolución en 72 horas $^{17}$. Por otra parte, la serie de Ballesteros \& Cols (1998) incluyó 12 casos con neumomediastino pediátrico y de ellos sólo un caso espontáneo’.

Hay otros casos aislados referidos como neumomediastino espontáneo, pero con historial de maniobras de Valsalva como desencadenantes (ejercicio intenso, extracción dental, jugar voleibol, crisis asmática, emesis de 5 días de evolución, exposición a un incendio, influenza, traqueomalacia), lo que los retira de la categoría',5,8,10,18,19,21,27,29.

En los casos colombianos, uno reportado por Campbell \& cols. (2016), otro por Morcillo y Vallejo (2015) y un tercero por Estrada \& cols. (2009), hubo desencadenantes que condujeron a maniobra de Valsalva (exposición a gasolina, motocross y levantar sacos de tierra, respectivamente) $)^{2,3,16}$. Por lo anterior, hace que realmente no se pueden ser considerardos como neumomediastinos espontáneos primarios.

En el caso del presente reporte no hubo ningún factor precipitante luego de una anamnesis detallada, una valoración física cuidadosa, exámenes de laboratorio e imagenológicos, lo que lo ubica como el primer caso colombiano reportado de neumomediastino espontáneo primario o idiopático.

La limitación más relevante en la revisión del tema radica en la dificultad conceptual del neumomediastino espontáneo primario, dado que muchos reportes en la literatura asimilan como iguales los casos con un desencadenante, incluso hay reportes adicionales en revisiones más amplias de neumomediastino pediátrico que no fueron citadas. 
Adicionalmente hasta la fecha no hay datos sobre alguna característica diferencial entre estos dos grupos y cualquier persona joven, especialmente entre la adolescencia y la adultez joven, pareciera estar en riesgo de presentar la patología.

\section{Conclusiones}

Se recomienda evaluar a todo niño escolar o adolescente con dolor torácico de inicio súbito, persistente y sin causa evidente, buscando el signo de Hamman palpando la región cervical en busca de crepitaciones, y realizando una radiografía de tórax para observar los signos de enfisema subcutáneo y de aire mediastinal; como indicadores de neumomediastino espontáneo. En los casos con desencadenantes $o$ enfermedades subyacentes aplica el mismo enfoque diagnóstico, debiendo ser más inquisitivos con menores de 6 años, presencia de neumotórax, y casos de sexo femenino. Es una medida prudente contar con el apoyo de cirugía pediátrica y neumología para el diagnóstico diferencial y el manejo de los casos.

\section{Referencias bibliográficas}

1. Girón Prieto MS, Ibáñez Godoy I. Spontaneous Pneumomediastinum in pediatric patient. Actual Medica. 2016; 101(798):138-9

2. Morcillo KL, Vallejo EL. Síndrome de Hamman: presentación de dolor torácico en un adolescente. Rev Colomb Cardiol. 2015; 22(1):62-5. http://dx.doi.org/10.1016/j.rccar.2014.12.002.

3. Estrada MC, Velásquez MI, Orrego M. Neumomediastino espontáneo: reporte de caso y revisión de la literatura. CES Med. 2009; 23(2):47-53.

4. Abbas PI, Akinkuotu AC, Peterson ML, Mazziotti M V. Spontaneous pneumomediastinum in the pediatric patient. Am J Surg. 2015; 210(6):1031-6

5. Olaciregui Echenique I, Plazaola Cortázar A, Uriz Monaut JJ, Korta Murua J. Enfisema subcutáneo y neumomediastino tras extracción dental. An Pediatr. 2014; 80(3):195-6

6. Álvarez Z C, Jadue T A, Rojas R F, Cerda C C, Ramírez V M, Cornejo S C. Neumomediastino espontáneo (síndrome de Hamman): Una enfermedad benigna mal diagnosticada. Rev Méd Chile. 2009; 137:1045-50.

7. Ballesteros S, García - Norniella B, Gracia A, Lagunilla L, Fernández - Menéndez J, Matesanz J. Neumomediastino no traumático en la edad pediátrica. Bol Pediatr. 1998; 38(164):1214.

8. Gasser CR, Pellaton R, Rochat CP. Pediatric spontaneous pneumomediastinum. Pediatr Emer Care. 2016;0(0):1-6

9. Yap V, Datta D. Spontaneous Pneumomediastinum. Chest
[Internet]. 2014; 145(3):265A. doi:10.1378/chest.1835826.

10. Gómez Tena G, Curto Simón B, Janer Subías E, Tello Martín A. Neumomediastino espontáneo: imás frecuente de lo que pensamos? Rev Pediatr Aten Primaria. 2016; 18:341-3.

11. Guasch Arriaga I, Staitie Gali A, Quintero Rivera J, López de Castro P, González Valencia A, Margelí Cervera V. Neumomediastino espontáneo y secundario no traumático: signos radiológicos ¿Como distinguirlos? Objetivo docente. SERAM. 2014; (S1148): $1-32$.

12. Bilkis MD, Monteverde E. Efecto Macklin en el neumomediastino espontáneo pediátrico. 2010; 108(2):33-6.

13. Murayama S. Spontaneous pneumomediastinum and Macklin effect: Overview and appearance on computed tomography. World J Radiol. 2014; 6(11):850

14. Lee $\mathrm{CY}, \mathrm{Wu} \mathrm{CC}$, Lin CY. Etiologies of spontaneous pneumomediastinum in children in middle Taiwan. Pediatr Pulmonol. 2010; 45(9):869-73.

15. Kin-sun W, Han-Meng W, Shen-Hao L, Chih-Yung C. Spontaneous pneumomediastinum. Ann Thorac Surg. 2004;78(2):711-3

16. Campbell S, Vargas SA, Gómez JM, Escobar AE, Muñoz JK. Síndrome de Hamman. Acta Med Colomb. 2016; 41:206-10

17. Crespo Marcos D, Iglesias Fernández C, Márquez de la Plata L, Panadero Carlavilla E, Vázquez López P. Neumomediastino espontáneo idiopático: a propósito de un caso. An Pediatría. 2006; 64(1):106-7

18. Tortajada-Girbés M, Moreno-Prat M, Ainsa-Laguna D, Mas S. Spontaneous pneumomediastinum and subcutaneous emphysema as a complication of asthma in children: case report and literature review. Ther Adv Respir Dis. 2016; 10(5):402-9.

19. Udupa S, Hameed T, Kovesi T. Pneumomediastinum and subcutaneous emphysema associated with pandemic (H1N1) influenza in three children. CMAJ. 2011; 183(2):220-2

20. Cho TJ, Kim H. Unusual presentation of spontaneous pneumomediastinum. Lung India. 2010; 27(4):239-41

21. Colavita L, Cuppari C, Pizzino MR, Sturiale M, Mondello B, Monaco F, et al. Pneumomediastinum, subcutaneous emphysema and pneumorrhachis in asthmatic children. J Biol Regul Homeost Agents. 2016; 30(2):585-8.

22. Kouritas VK, Papagiannopoulos K, Lazaridis G, Baka S, Mpoukovinas I, Karavasilis V, et al. Pneumomediastinum. J Thorac Dis. 2015; 7(Suppl 1):S44-9

23. Ammar A, Ibrahim F. Spontaneous pneumomediastinum in children and adolescents Spontaneous pneumomediastinum in children and adolescents. Post TW, ed. UpToDate. Waltham, MA: UpToDate Inc (Consultado febrero 2018).

24. Zachariah S, Gharahbaghian L, Perera P, Joshi N. Spontaneous pneumomediastinum on bedside ultrasound: Case report and review of the literature. West Jour Emerg Med. 2015; 16(2):321-4.

25. Ng, L; Saul, T; Lewiss R. Sonographic evidence of spontaneous pneumomediastinum. Am J Emerg Med. 2013; 31(2): 462.e3-462. e4

26. Almeida V, Costa R, Godinho S. Neumomediastino espontáneo en un primer episodio de disnea. Rev Portales Medicos 2017; 2-6.

27. Nair B, Surendran S, Brar T. Spontaneous subcutaneous emphysema and pneumomediastinum in a child with bronchial asthma. Ann Niger Med. 2015; 9(1):30.

28. Londoño MP, Ávila Arenas LM. Neumomediastino espontáneo: revisión de tema. Univ medica. 2017; 58(4):1-7.

29. Dewulf, J., Van Daele, S., De Baets, F. Recurrent spontaneous pneumomediastinum in a child with tracheomalacia. Pediatric Pulmonology, 2016, 10-12. http://doi.org/10.1002/ppul.23624

30. Ansótegui Barrera, E., Mancheño Franch, N., Vera-Sempere, F., Padilla Alarcón, J. Linfangioleiomiomatosis. Archivos de Bronconeumología, 2011. 47(2), 85-93. http://doi.org/10.1016/j. arbres.2010.08.008 